\title{
Eosinophilic pancreatitis: Three case reports and literature review
}

\author{
LIN TIAN $^{1}$, PENG FU ${ }^{2}$, XIANGHUI DONG ${ }^{1}$, JIPING QI ${ }^{1}$ and HONG ZHU ${ }^{1}$ \\ Departments of ${ }^{1}$ Pathology and ${ }^{2}$ Nuclear Medicine, The First Affiliated Hospital of \\ Harbin Medical University, Harbin, Heilongjiang 150001, P.R. China
}

Received October 6, 2015; Accepted January 20, 2016

DOI: $10.3892 / \mathrm{mco} .2016 .760$

\begin{abstract}
Eosinophilic pancreatitis (EP) is a rare form of chronic pancreatitis characterized by localized or diffuse eosinophilic infiltration of the pancreas and elevated serum immunoglobulin E levels. EP is difficult to distinguish from pancreatic cancer on the basis of clinical symptoms and the results of auxiliary examination alone. A retrospective analysis of the clinicopathological characteristics and laboratory, imaging, and pathology results of 3 patients with $\mathrm{EP}$, who were initially diagnosed with pancreatic malignancy, was performed. EP is an allergic disease with non-specific clinical manifestations that is difficult to distinguish from pancreatic cancer based exclusively on clinical symptoms and auxiliary examination, resulting in the need for invasive procedures to confirm the diagnosis. An increase in the eosinophil count in the peripheral blood and pathological examination are essential for the diagnosis of EP.
\end{abstract}

\section{Introduction}

Eosinophilic pancreatitis (EP) is a rare form of chronic pancreatitis characterized by localized or diffuse eosinophilic infiltration of the pancreas and elevated serum immunoglobulin (Ig) E levels. EP is difficult to distinguish from pancreatic cancer on the basis of clinical symptoms and the results of auxiliary examination alone. Therefore, the majority of the patients undergo pancreatic surgery to confirm the diagnosis. We herein describe 3 cases of EP patients who underwent pancreatoduodenectomy due to a preoperative diagnosis of cancer of the pancreatic head or choledochal cancer. The clinicopathological characteristics or these EP patients were investigated in the present study to improve our understanding of this disease and reduce the rates of misdiagnosis and missed diagnosis.

Correspondence to: Dr Hong Zhu or Professor Jiping Qi, Department of Pathology, The First Affiliated Hospital of Harbin Medical University, 23 Youzheng Street, Nangang, Harbin, Heilongjiang 150001, P.R. China

E-mail: hong.zhu484@gmail.com

E-mail: bless88775469@163.com

Key words: eosinophilic pancreatitis, eosinophil infiltration

\section{Case report}

Case 1. A 39-year-old man was admitted to The First Affiliated Hospital of Harbin Medical University (Harbin, China) in February, 2006 with a 2-week history of jaundice. The patient denied drug and alcohol use, and had a negative history of allergies. The physical examination was unremarkable, expect for the yellowish skin and sclerae. The laboratory findings included a percentage of peripheral blood eosinophils of $11 \%$, total bilirubin (TB) of $147.5 \mu \mathrm{mol} / 1$ and direct bilirubin (DB) of $87.4 \mu \mathrm{mol} / 1$. The B-ultrasound and computed tomography (CT) results revealed diffuse enlargement of the pancreatic head, expansion of the intrahepatic and extrahepatic bile ducts, and pancreatic duct dilatation. The patient was diagnosed with pancreatic head cancer and underwent pancreatoduodenectomy. Postoperatively, the pancreatic specimens were enlarged and had a diffusely hard texture on cross section. A gray mass measuring $2 \times 2.3 \times 1.5 \mathrm{~cm}$ with indistinct boundaries appeared to constrict the pancreatic duct and common bile duct. On microscopic examination, there was diffuse inflammatory cell infiltration of the pancreatic duct, acini and interstitium, mostly by eosinophils and a few lymphocytes and plasma cells (Fig. 1A, C and D). Fibrous connective tissue hyperplasia and collagenation were also observed (Fig. 1A and B). The patient tested negative for parasites, rheumatism and autoimmune antibodies, which excluded the possibility of autoimmune pancreatitis and parasitic infection. A diagnosis of EP was made. The patient presented 2 months after surgery with jaundice, TB of $116.5 \mu \mathrm{mol} / 1, \mathrm{DB}$ of $82.6 \mu \mathrm{mol} / 1$, elevated serum IgE and normal IgG levels. The patient was treated with $40 \mathrm{mg} / \mathrm{day}$ prednisone for 6 days, after which the jaundice subsided.

Case 2. A 46-year-old man was admitted to our hospital in June, 2010 with an 8-day history of jaundice and mild abdominal pain. The patient had a history of asthma and denied alcohol and medication use. The laboratory findings included a percentage of peripheral blood eosinophils of $10 \%$, TB of $127.2 \mu \mathrm{mol} / 1$ and DB of $77.6 \mu \mathrm{mol} / 1$. The B-ultrasound and CT results revealed diffuse enlargement of the pancreas and a low-density shadow in the pancreatic head. Endoscopic retrograde cholangiopancreatography (ERCP) revealed stenosis of the distal bile duct and pancreatic duct at the head of the pancreas, and pancreatic duct dilatation adjacent to the area of stenosis. The proposed diagnosis was pancreatic head carcinoma and the patient underwent pancreatoduodenectomy. Postoperatively, the pancreatic 

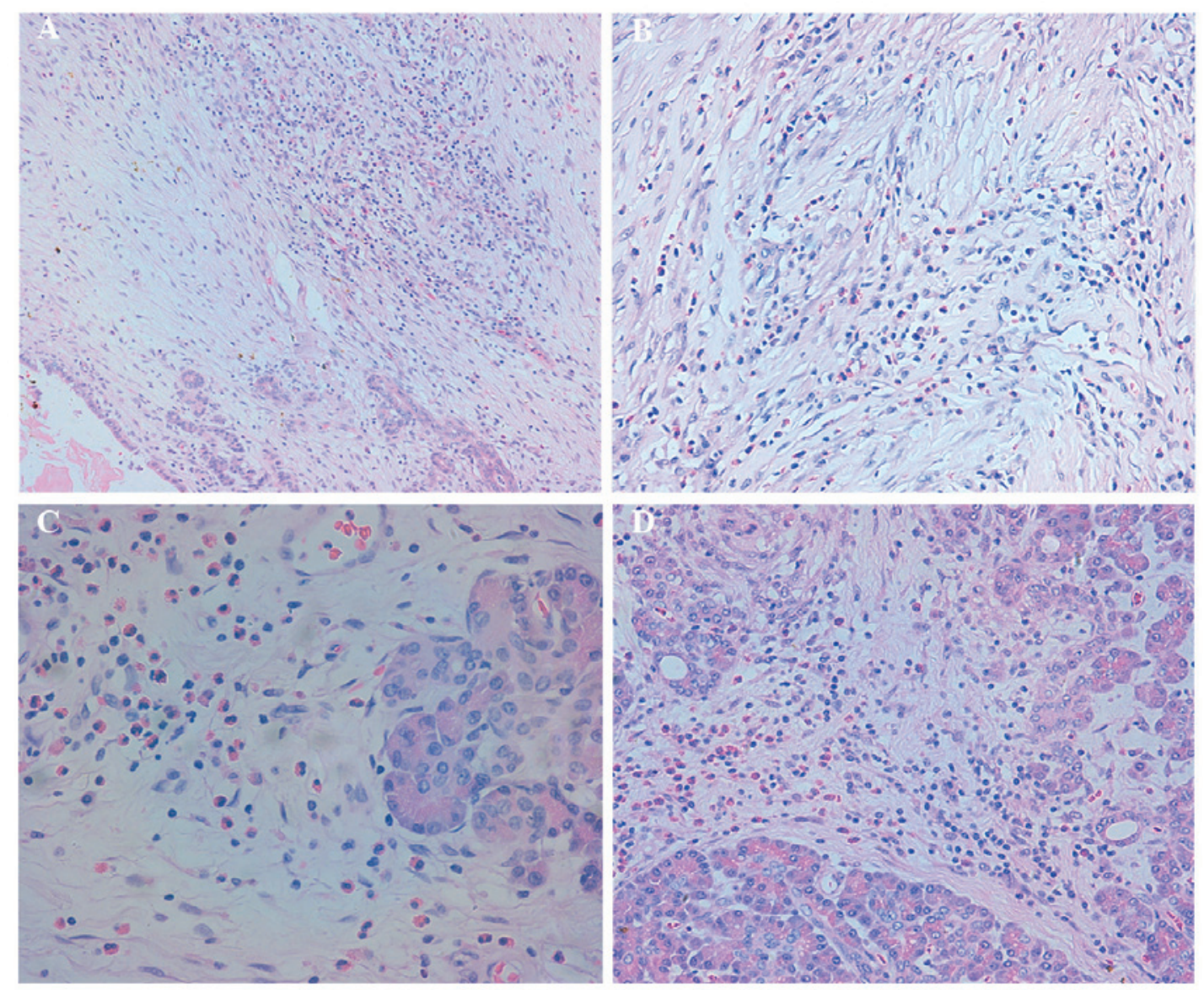

Figure 1. (A, C and D) Infiltration of the pancreatic duct, acini and interstitium by inflammatory cells, mainly consisting of eosinophils and a few lymphocytes and plasma cells. (A and B) Fibrous connective tissue hyperplasia with collagenation was also obsrved.

specimens displayed an enlarged pancreatic head, with a diffusely hard texture on cross section. A gray mass measuring $2 \times 2.3 \times 1.5 \mathrm{~cm}$ with indistinct boundaries was detected, which appeared to constrict the common bile duct. On microscopic examination, there was a diffuse inflammatory cell infiltration of the pancreatic parenchyma and interstitium, mostly consisting of eosinophils and a few lymphocytes and plasma cells. Fibrous connective tissue hyperplasia and collagenation were also observed. The patient tested negative for parasites, rheumatism and autoimmune antibodies, which excluded the possibility of autoimmune pancreatitis and parasite infection. A diagnosis of EP was made.

Case 3. A 41-year-old woman was admitted to our institution in June, 2013 with a 4-day history of abdominal pain, fatigue, loss of appetite, weight loss, nausea, vomiting and jaundice. The patient denied alcohol and medication use and had a negative history of allergic diseases. The laboratory findings included a peripheral blood eosinophil percentage of 3\%, TB of $127.2 \mu \mathrm{mol} / 1$ and DB of $97.6 \mu \mathrm{mol} / 1$. The CT scan revealed intrahepatic and extrahepatic bile duct dilatation and delayed enhancement of the lower end of the common bile duct, A diagnosis of choledochal cancer was made and the patient underwent pancreatoduodenectomy. Postoperatively, the pancreatic specimens exhibited stenosis of the intrapancreatic portion of the common bile duct, with a smooth mucosa and absence of masses, surrounded by hardened pancreatic tissue exhibiting a coarse granular texture on cross section. Constriction of the common bile duct was observed. On microscopic examination, there was an inflammatory cell infiltration of the pancreatic parenchyma and interstitium, mostly by eosinophils and a few lymphocytes and plasma cells. Fibrous connective tissue hyperplasia and collagenation were also observed. The $\operatorname{IgE}$ and $\operatorname{IgG}$ levels were within the normal range and the patient tested negative for parasites, rheumatism and autoimmune antibodies. A diagnosis of EP was made.

\section{Discussion}

EP is a rare form of chronic pancreatitis characterized by localized or diffuse eosinophilic infiltration of the pancreas, accompanied by an increase in IgE levels. Abraham et al (1) analyzed 3,375 patients with pancreatic disease; among those, 26 exhibited eosinophilic infiltration of the pancreas, of whom only 3 patients were diagnosed with EP. This disease has been reported in animals, including nematode-infected horses, Toxocara canis-infected dogs, mice injected with purified scorpion venom, horses with T-cell lymphoma and ferrets with Hodgkin's disease (2-7).

A diagnosis of EP is often made following exclusion of parasitic infection in patients presenting with abdominal pain, vomiting, diarrhea and other gastrointestinal symptoms, who exhibit marked eosinophilic infiltration in pathological samples (despite the lack of a standard for eosinophil counts) and no organ involvement outside the digestive system $(8,9)$. 
The clinical symptoms and characteristics of the patients during the course of the EP are as follows: A peripheral eosinophil count of $>1.5 \times 10^{9}$ for $>6$ months; a history of rhinitis, bronchial asthma and other allergic diseases; eosinophilic infiltration of other organs, such as the heart, skin and gastrointestinal tract; exclusion of other causes of eosinophilia, such as leukemia and parasitic infections; and a diagnosis of hypereosinophilic syndrome according to the international standards (10). An increase in blood eosinophil count may not be present in patients with other diseases associated with eosinophilia during the early stages; therefore, close observation is mandatory.

Pancreatic diseases associated with eosinophilia include EP, pancreatic cancer, autoimmune pancreatitis and alcoholic pancreatitis. EP and autoimmune pancreatitis are easily misdiagnosed as pancreatic cancer and are often treated surgically due to the similarity in their clinical symptoms and imaging findings, such as pancreatic enlargement; furthermore, a proportion of cases of pancreatic cancer are associated with eosinophilia (11). The differential diagnosis mainly depends on the histopathological findings and the measurement of serum tumor marker levels, as well as differences in the results of ERCP, history of asthma and allergy, increased IgE and IgG levels, and the effectiveness of glucocorticoid treatment.

EP is difficult to distinguish from autoimmune pancreatitis, which exhibits similar clinical manifestations and CT and ERCP results compared with those of EP. The main characteristics that may be helpful for the differential diagnosis of these two conditions are as follows $(1,12)$ : i) The histopathological changes of EP include diffuse eosinophilic infiltration of the pancreatic ducts, acini and interstitium, accompanied with eosinophilic phlebitis; the inflammatory infiltrate of the pancreas is mainly composed by eosinophils, or may be exclusively eosinophilic in a few cases, with localized high-density infiltration observed in cases with pancreatic pseudocysts; ii) the lesions of autoimmune pancreatitis are mainly infiltrated by lymphocytes rather than eosinophils; iii) EP is associated with an increase in IgE levels in the peripheral blood, whereas patients with autoimmune pancreatitis exhibit elevated IgG4 levels; iv) autoimmune pancreatitis patients generally test positive for autoimmune and antinuclear antibodies; and v) autoimmune pancreatitis patients exhibit an evenly enlarged (sausage-like) pancreas, rather than enlargement of the pancreatic head or tail.

The morphological manifestations in the 3 patients described in the present study were consistent with those of EP. Autoimmune pancreatitis and parasitic infections were excluded based on the postoperative pathological changes and additional clinical history and laboratory examination, including a negative history of alcohol and medication use, leading to a diagnosis of EP. The 3 patients did not meet the diagnostic criteria of eosinophilic gastroenteritis or eosinophilic syndrome; however, the increased eosinophil count and IgE levels in the peripheral blood indicate the need for close observation, considering that a proportion of cases of eosinophilia combined with other diseases do not exhibit an elevated eosinophil count in the early stages of the disease. Although a diagnosis of EP was made in the 3 cases based on the results of postoperative pathology, EP was not suspected preoperatively, underscoring the need to improve our knowledge of EP to prevent misdiagnosis. In our cases, the medical history was incomplete and eosinophilia in routine blood tests was overlooked, with no preoperative detection of autoantibodies or increased serum IgE levels, which led to misdiagnosing EP for cancer. In patients with chronic inflammation of the pancreas, a frozen section biopsy of the mass during surgery may prevent misdiagnosis and unnecessary surgical interventions.

A diagnosis of EP remains difficult for the following reasons: i) Low incidence and, consequently, limited information in the available literature; ii) no specific clinical symptoms; iii) high technical requirements for diagnosis, as neither IgE measurements nor endoscopic ultrasound-guided pancreatic biopsy may be performed in a General Hospital; and iv) the presence of tumor-like changes, which may lead to a misdiagnosis of pancreatic cancer.

In summary, EP is a rare disease that is easily misdiagnosed in clinical practice. This is partly due to the increasing incidence of pancreatic cancer and autoimmune pancreatitis, combined with a difficulty in differential diagnosis among these three conditions. Laboratory tests, including eosinophil counts and IgE levels, combined with CT and ERCP, as well as pathological examination of the biopsy specimens, are necessary for the diagnosis of EP. Glucocorticoid diagnostic treatment may be used when these tests are not possible, with rapid shrinkage of the pancreatic mass and a decrease of the eosinophil count suggesting EP. Non-invasive treatments that preserve pancreatic function are preferred, as they minimize the impact on the patients' quality of life. An accurate diagnosis of EP is crucial, as it is considered to be a hypereosinophilic syndrome. Surgical intervention may be used to alleviate the biliary duct obstruction and resect the pancreatic head lesion; however, other pathological changes of the pancreatic body and tail, as well as the involvement of other organs, such as the skin, liver, spleen, lymph nodes, cardiovascular system and respiratory system, require close follow-up.

\section{Acknowledgements}

The present study was supported by the Natural Science Foundation of Heilongjiang Province (grant no. H201445 to Dr Hong Zhu).

\section{References}

1. Abraham SC, Leach S, Yeo CJ, Cameron JL, Murakata LA, Boitnott JK, Albores-Saavedra J and Hruban RH: Eosinophilic pancreatitis and increased eosinophils in the pancreas. Am J Surg Pathol 27: 334-342, 2003.

2. Breider MA, Kiely RG and Edwards JF: Chronic eosinophilic pancreatitis and ulcerative colitis in a horse. J Am Vet Assoc 186: 809-811, 1985.

3. Petty DP, Lange AL, Verster A and Hattingh J: Necropsies of eight horses infected with Strongylus equinus and Strongylus edentatus. J S AfrVet Assoc 63: 66-69, 1992.

4. Hayden DW and Kruiningen HJ: Experimentally induced canine toxocariasis: Laboratory examinations and pathologic changes, with emphasis on the gastrointestinal tract. Am J Vet Res 36: 1605-11614, 1975.

5. Novaes G, Cardozo Cde C, Costa NM, de Falco CN, de Carvalho $\mathrm{MH}$ and de Queiroz AC: Experimental chronic interstitial pancreatitis induced by scorpion toxin in rats. Arq Gastroenterol 27: 187-190, 1990.

6. La Perle KM, Peircy RJ,Long JF and Blomme EA: Multisystemic, eosinophilic, epitheliotropic disease with intestinal lymphosarcoma in a horse. Vet Pathol 35: 144-146, 1998. 
7. Blomme EA, Foy SH, Chappell KH and La Perle KM: Hypereosinophilic syndrome with Hodgkin's-like lymphoma in a ferret. J Comp Pathol 120: 211-217, 1999.

8. Le Connie D and Nguyen H: Eosinophilic gastroenteritis and pancreatitis: A case report and review of the literature. South Med J 97: 905-906, 2004

9. Javid Bhat K, Bhat S, Dutt K, Gupta S and Jeelani Samoon H: Chronic diarrhea, eosinophilic ascites, acute pancreatitis and deep venous thrombosis: A case report. Caspian J Intern Med 5: $182-185,2014$.

10. Valent P, Klion AD, Horny HP, Roufosse F, Gotlib J, Weller PF, Hellmann A, Metzgeroth G, Leiferman KM, Arock M, et al: Contemporary consensus proposal on criteria and classification of eosinophilic disorders and related syndromes. J Allergy Clin Immunol 130: 607-612, 2012.
11. Euscher E, Vaswani K and Frankel W: Eosinophilic pancreatitis: A rare entity that can mimic a pancreatic neoplasm. Ann Diagn Pathol 4, 379-385, 2000

12. Shimosegawa T, Chari ST, Frulloni L, Kamisawa T, Kawa S, Mino-Kenudson M, Kim MH, Klöppel G, Lerch MM, Löhr M, et al: International consensus diagnostic criteria for autoimmune pancreatitis: Guidelines of the International Association of Pancreatology. Pancreas 40: 352-358, 2011. 